\title{
Computer aided detection and diagnosis in radiology
}

\author{
E. Kotter • M. Langer
}

Received: 25 November 2010 /Revised: 13 December 2010 /Accepted: 15 December 2010 /Published online: 11 January 2011

(C) European Society of Radiology 2011

During the past two decades, the volume of image data produced within Radiology Departments has grown continuously, with a doubling time of approximately 2 years. This increase is mainly due to the growing demand for high resolution sectional imaging. It can be foreseen that introduction of functional imaging techniques into clinical routine will contribute to further increases in data production in radiology. However, the number of radiologists has not been growing in parallel. Consequently, the productivity in terms of number of images to be analysed in a given time span per radiologist has increased substantially in the past few years and will be further growing in the future.

In the main, this increased productivity within Radiology Departments has been made possible with the introduction of new modes of viewing (digital workstations, stack view of images, fast multiplanar reconstructions), fast retrieval of images (picture archiving and communication systems), better access to clinical information (electronic patient records), and reporting improvements (radiology information systems, speech recognition). Despite such improvements, there will be yet further demands on radiologists' time as complex time-consuming techniques become more widely adopted (eg CT colonography screening). Thus there is even more need to help productivity in the future.

E. Kotter $(\bowtie) \cdot$ M. Langer

Abteilung Röntgendiagnostik,

Hugstetterstrasse 55, Universitätsklinikum Freiburg,

D-79106 Freiburg, Germany

e-mail: elmar.kotter@uniklinik-freiburg.de
Potential solutions include new visualisation techniques for some specific examinations techniques, as in CT colonography and mammography [1]. On a broader basis, the introduction of emerging techniques of computer aided detection (CAD) into the radiologist's workflow, should eventually increase the speed of interpretation without negative effects on accuracy, in the best case may even have a positive effect on accuracy.

One field where CAD has been well evaluated over the last decade is the detection of lung nodules on CT studies of the thorax. Most studies have shown an improvement in the detection of lung nodules when CAD is used. This seems to be independent of the CT technique used or the experience of the human reader. While CAD for lung nodule detection and mammography [2] has been most extensively evaluated, CAD systems for other applications (virtual colonography, detection of lymph nodes, detection of hepatic metastases) have emerged during the last years. Despite the proven advantages of CAD systems, they have not yet made their way into routine clinical use in many centres. One main reason for this is the way CAD systems are designed today.

Virtually all available CAD systems rely on specialised standalone CAD-workstations, providing not only CAD, but also other tools like segmentation, volumetry of lung nodules, and the ability to (semi-) automatically compare nodules on sequential examinations (Fig. 1). Despite those advantages, there are many inconveniences to use specialised workstations. From a technical viewpoint, the need to route images not only to the PACS but also to the CAD-workstation is a major inconvenience, especially as several specialised workstations may be needed, each for a 
Fig. 1 Results of lung nodule CAD with automatic detection, volumetry, and determination of growth rate between two consecutive examinations. Clicking the points in the overview images in the upper part of the screen shows detailed information about the nodules in the lower part of the screen

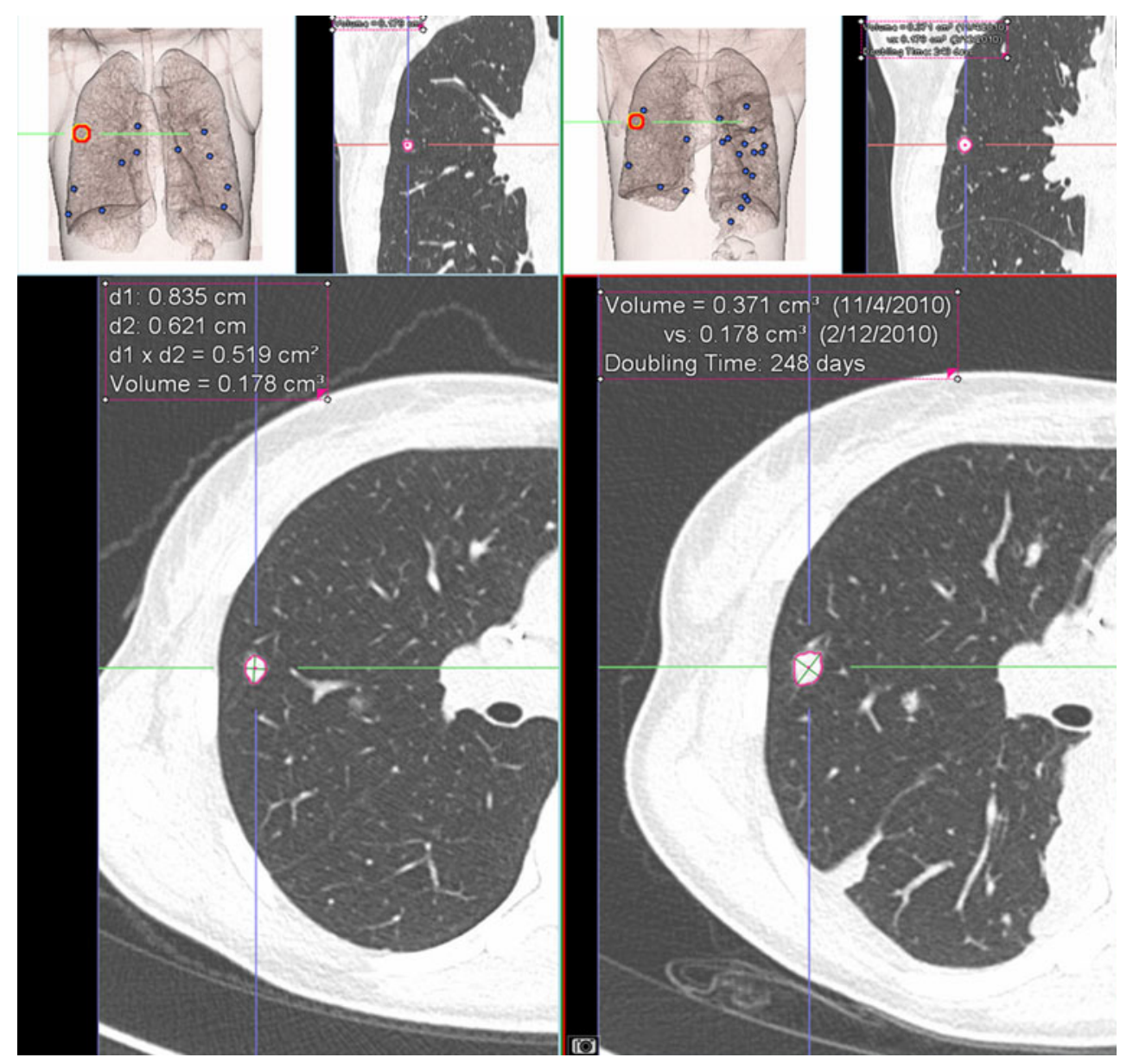

different specialised examination (lung nodule CAD, CT colonoscopy $\mathrm{CAD}$, and others). This would require either massive routing to all workstations or the need to maintain complex routing protocols for more intelligent routing.

The main inconvenience in using specialised CAD workstations is the need for the radiologist to work with at least two different workstations when interpreting studies using CAD. In some cases (like additional RECIST workup), the use of a third workstation may even be needed. The need to use different workstations may force the radiologist to physically move from one place to another; this may result in the CAD workstation not being used for all cases, even where appropriate. This issue can be overcome by using CAD servers with net clients installed on the radiologist's workstation. But, more importantly, the use of different CAD-systems (usually from different manufacturers) also confronts the radiologist with completely different and unfamiliar user interfaces.

When considering a radiologist's diagnostic work, accuracy certainly is the most important feature, but one must also take into account efficiency of a single radiologist's workload within a given time. The ideal is to 'produce' a maximum of correct diagnoses per time unit.
Ideal CAD systems should increase the accuracy of radiologists and/or accelerate their workflow.

Older research on CAD systems examined only the ability of CAD systems to enhance accuracy (usually by increasing the sensitivity of the diagnostic system, sometimes at the cost of a somewhat decreased specificity), without taking into account the time it takes to achieve the diagnosis. Only recently have investigators also included the aspect of reading time into their study designs.

Beyer et al. [3] compare "sensitivity and reading time for the use of CAD of pulmonary nodules at MDCT as concurrent or second reader". In comparing the analysis of 50 chest MDCT examinations by four radiologist using CAD either as a concurrent reader or using CAD as second reader, they found that sensitivity of reading with CAD as second reader was significantly higher than reading without $\mathrm{CAD}$ and reading with $\mathrm{CAD}$ as concurrent reader, while there was no significant difference between reading with $\mathrm{CAD}$ as concurrent reader or reading without CAD. Reading time was significantly shorter for CAD as concurrent reader compared to reading without $\mathrm{CAD}$ or CAD as second reader. Thus CAD could either speed up reading of chest $\mathrm{CT}$ cases without relevant loss of 
sensitivity, or it increased sensitivity at the cost of longer reading times when used as second reader. In days of increasing competition between departments such choices become difficult on economic grounds.

Roos et al. [4] in a very original approach, evaluated "radiologists performance and reading time with incremental CAD assistance" in "CAD of lung nodules in CT". They posed the question whether the threshold for lesion inclusion within CAD systems influences performance of the complete diagnostic system, consisting of both the radiologist and the CAD system; they also analysed temporal behaviour for acceptance and rejection of lung nodules and non nodules presented as possible nodules by the CAD system. With the particular setup of the CAD System presenting to the human reader first the lesions with a high probability of being true positives, they were able to show that (1) radiologists derive variable benefits from $\mathrm{CAD}$, (2) that $\mathrm{CAD}$ has the ability to equalise performance among readers by reducing individual detection errors of lung nodules on chest CT and (3) that analysis of CAD-dependent temporal variations in performance may facilitate the establishment of optimised thresholds for the duration of CAD interaction.

Both articles show that many questions need to be discussed concerning CAD. Beyer et al. [3] show how the order in which human reading and/or CAD is used influence accuracy and speed of interpretation. In the future there might be technological solution that allow the usage of $\mathrm{CAD}$ as a second reader without a time penalty; this, however necessitates the tight integration of the CAD system within the radiologists daily workplace, presenting the radiologist first (without interruption) the images only, followed by the images with an overlay of CAD images. The addition of the ability to integrate the results of previous examinations automatically (follow-up of lesions that have been identified previously) might further improve the efficacy of the system.
From a user's perspective, the worst way to 'integrate' CAD systems, is to run them on specialised workstations. The second best solution is to have a simple interface between the workstation and the CAD application, with transmission of patient data to the CAD application. The best integration is achieved when integration is transparent for the user, i.e. the workstations interface, displaying seamlessly results of the CAD application running on some server in the background.

Introduction of CAD techniques into the daily clinical workflow of radiologists is one of the great challenges for the upcoming decade. As shown by the two papers cited above, this will be a complex task with many open questions, not only from the technical side, which needs strong input from PACS and CAD vendors, but also from the exact place where different CAD methods should be introduced into the workflow. We hope to see more work on these questions in European Radiology in the future.

\section{References}

1. Mang T, Kolligs FT, Schaefer C, Reiser MF, Graser A (2010) Comparison of diagnostic accuracy and interpretation times for a standard and an advanced 3D visualisation technique in $\mathrm{CT}$ colonography. Eur Radiol. doi:10.1007/s00330-010-1953-x

2. Samulski M, Hupse R, Boetes C, Mus RD, den Heeten GJ, Karssemeijer N (2010) Using computer-aided detection in mammography as a decision support. Eur Radiol 20:2323-2330

3. Beyer F, Zierott L, Fallenberg EM, Juergens KU, Stoeckel J, Heindel W, Wormanns D (2007) Comparison of sensitivity and reading time for the use of computer-aided detection (CAD) of pulmonary nodules at MDCT as concurrent or second reader. Eur Radiol 17:2941-2947

4. Roos JE, Paik D, Olsen D, Liu EG, Chow LC, Leung AN, Mindelzun R, Choudhury KR, Naidich DP, Napel S, Rubin GD (2010) Computer-aided detection (CAD) of lung nodules in CT scans: radiologist performance and reading time with incremental CAD assistance. Eur Radiol 20:549-557 